\title{
Corrigendum
}

\section{Glucocorticoid resistance in T-lineage acute lymphoblastic leukaemia is associated with a proliferative metabolism}

\author{
AH Beesley, MJ Firth, J Ford, RE Weller, JR Freitas, KU Perera and UR Kees
}

British Journal of Cancer (2010) 102, 1200. doi:I0.1038/sj.bjc.6605632 www.bjcancer.com

(C) 2010 Cancer Research UK

Correction to: British Journal of Cancer (2009) 100, 1926-1936. doi:10.1038/sj.bjc.6605072

In reference to Figure $4 \mathrm{D}$, the drug quercetin is clearly cytotoxic as a single agent in the ALL-SIL cell line, but its effect in combination with dexamethasone (DEX) is not synergistic as originally quoted. Rather, the combination of quercetin and DEX resulted in a small, though non-significant reduction in cytotoxicity. This is in contrast to resveratrol (Figure 4E), which demonstrated synergistic cytotoxicity in combination with DEX. This difference in the two agents is reflected in their connectivity map (CMAP) enrichment scores in Table 3, with quercetin showing a positive enrichment in contrast to the negative scores for resveratrol, LY-294002 and rapamycin. Both quercetin and resveratrol are polyphenols that activate the key metabolic regulator AMPK, but the results suggest that they are mechanistically different and are unlikely to be equally efficacious in combinatorial therapy with glucocorticoids in T-cell acute lymphoblastic leukaemia (T-ALL). Figure 4D and Table 3 from the original paper are reproduced for reference, below.

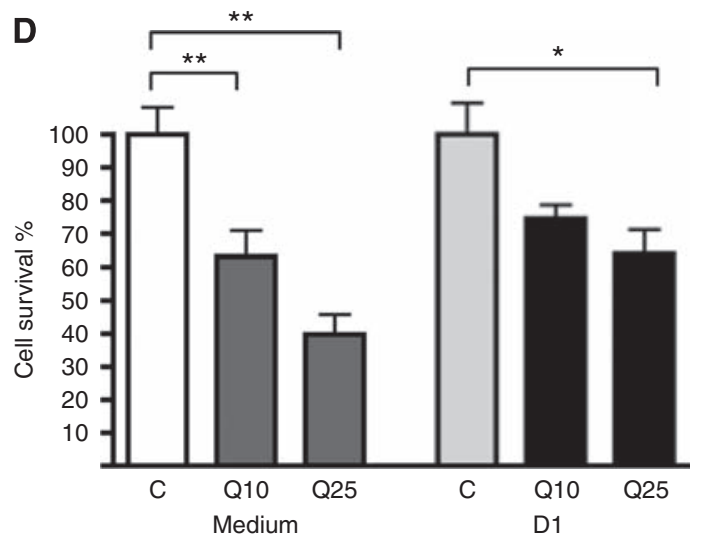

Figure 4 Synergy of CMAP-identified drugs with DEX in T-ALL cell lines. Graphs show cell survival following $48 \mathrm{~h}$ incubation. Comparisons were made in each data set to the respective control condition, which was set to 100\%. Vehicle control (C) and the following drug treatments were analysed: (D) $10 \mathrm{~mm}$ (Q I0), $25 \mathrm{~mm}$ (Q25) quercetin and I mM DEX (DI) in ALL-SIL.

Table 3 CMAP drugs significantly associated with MPRED- and DEX-resistance gene signatures in T-ALL cell lines

\begin{tabular}{|c|c|c|c|c|c|c|c|}
\hline \multicolumn{4}{|c|}{ MPRED } & \multicolumn{4}{|c|}{ DEX } \\
\hline Drug & Mechanism of action & Enrichment & $P$-value & Drug & Mechanism of action & Enrichment & $P$-value \\
\hline Trichostatin A & HDAC inhibitor & -0.423 & 0.018 & Geldenamycin & HSP9O inhibitor & 0.655 & 0.004 \\
\hline Carbamazepine & Antiepileptic & -0.792 & 0.020 & LY-294002 & AKT/PI3K inhibitor & -0.397 & 0.005 \\
\hline$W-13$ & Calmodulin antagonist & 0.903 & 0.021 & Sodium phenylbutyrate & HDAC inhibitor & -0.564 & 0.014 \\
\hline Blebbistatin & Myosin II inhibitor & -0.896 & 0.023 & Quercetin & ROS scavenger/antioxidant & 0.903 & 0.019 \\
\hline Rapamycin & mTOR inhibitor & -0.441 & 0.031 & Fludrocortisone & Steroid & 0.857 & 0.042 \\
\hline Indomethacin & Cyclo-oxygenase inhibitor & 0.662 & 0.031 & Deferoxamine & Hypoxia mimetic & -0.717 & 0.049 \\
\hline Quercetin & ROS scavenger/antioxidant & 0.876 & 0.033 & & & & \\
\hline Y-27632 & Rho-kinase inhibitor & -0.872 & 0.036 & & & & \\
\hline Tamoxifen & Oestrogen-receptor blocker & 0.740 & 0.039 & & & & \\
\hline Resveratrol & ROS scavenger/antioxidant & -0.583 & 0.040 & & & & \\
\hline
\end{tabular}

Data indicate the CMAP-calculated enrichment score and permuted P-value for association with GC expression profiles. 